\title{
Subcutaneous NPH Insulin for Severe Hypertriglyceridemia in a Pregnant Patient with Type V Hyperlipoproteinemia: a Case Report
}

\author{
Güven Barış Cansu, Nusret Yılmaz, Hasan Altunbaş, Mustafa Kemal Balcı, Ramazan Sarı \\ Department of Endocrinology and Metabolism, Faculty of Medicine, Akdeniz University, Antalya, Turkey
}

\begin{abstract}
An increase in triglyceride levels in familial hyperlipidemia during pregnancy has been reported. Severe hypertriglyceridemia can lead to complications such as acute pancreatitis, preeclampsia, maternal and fetal complications. Because of the teratogenic effects associated with fibrate therapy in pregnancy, alternative treatment strategies such as insulin as a rapid and potent activator of lipoprotein lipase are required during pregnancy. We report a case of hypertriglyceridemia in a 33-year-old pregnant woman in whom treatment with merely single one time administration of Neutral Protamine Hagedorn insulin was accompanied by a reduction in the serum triglyceride level; to the best of our knowledge, this has never been reported in the literature. Her triglyceride level was $3616 \mathrm{mg} / \mathrm{dL}$ before insulin treatment and $1246 \mathrm{mg} / \mathrm{dL}$ after insulin treatment. Although this regimen was used safely and effectively in our patient, comprehensive studies are required to evaluate the effectiveness and safety of subcutaneously intermediate-acting Neutral Protamine Hagedorn insulin for the treatment of severe hypertriglyceridemia in non-diabetic pregnant women.
\end{abstract}

Key Words: Pregnancy, hypertriglyceridemia, insulin

Received: 19.12.2011 Accepted: 28.03.2012

\section{Introduction}

The primary causes of severe hypertriglyceridemia include lipoprotein lipase deficiency, apolipoprotein C-II deficiency, the presence of an apolipoprotein E2 allele, familial hypertriglyceridemia or familial combined hyperlipidemia $(1,2)$. The exacerbation of triglyceride levels in familial hyperlipidemia during pregnancy is multifactorial. An increase in estrogen levels during pregnancy results in increased hepatic very low-density lipoprotein (VLDL) synthesis (3). Lipoprotein lipase activity is also reduced in pregnancy. Furthermore, human placental lactogen increases adipose tissue lipolysis and serum-free fatty acid levels, thus facilitating the availability of substrates to the liver for triglyceride synthesis. The combination of these phenomena result in an increase in VLDL production coupled with reduced triglyceride clearance, leading to a net increase in serum triglyceride levels (4).

The main treatment modality for hypertriglyceridemia during pregnancy includes dietary restriction of fat, heparin, insulin, lipid-lowering medications (mainly fibrates) and plasmapheresis (5). The rationale for using insulin in the treatment of severe hypertriglyceridemia is based on the fact that insulin is a rapid and potent activator of lipoprotein lipase, most likely acting by stimulating its synthesis (6). Data regarding the use of insulin or the management of severe hypertriglyceridemia in non-diabetic patients are very limited. We report a case of hypertriglyceridemia in a 33-year-old pregnant woman in whom treatment with a single daily dose of Neu- tral Protamine Hagedorn (NPH) insulin was accompanied by a reduction in the serum triglyceride level, which, according to our knowledge, has never been reported in the literature.

\section{Case Report}

A 33-year-old multigravida woman at 10 weeks of gestation was referred to the Department of Endocrinology at Akdeniz University Hospital in Antalya for hypertriglyceridemia and the use of fenofibrate. She denied any alcohol use, recently prescribed new medications and no history of diabetes mellitus or pancreatitis. Her first pregnancy, in 2006, was uneventful and she delivered a healthy female child, weighing 3000 grams. Hypertriglyceridemia was diagnosed in 2008 and $267 \mathrm{mg}$ fenofibrate daily had been taken for three years. Her family history was positive for lipid disorders; the patient's father has diabetes mellitus and severe hypertriglyceridemia and her sister has hypertriglyceridemia. Her mother and brother have no history of hypertriglyceridemia.

The physical examination revealed a temperature of $36.6^{\circ} \mathrm{C}$, blood pressure of $120 / 70 \mathrm{mmHg}$, pulse of $94 / \mathrm{min}$, respiratory rate of $16 / \mathrm{min}$ and there were no abnormal findings in the respiratory, cardiovascular and nervous systems. Her height was $154 \mathrm{~cm}$, weight was $63 \mathrm{~kg}$ and body mass index was $26.6 \mathrm{~kg} / \mathrm{m}^{2}$. She had no clinical signs suggestive of hyperlipidemia. Obstetric examination was normal and ultrasound examination revealed that fetal growth was appropriate for gestational age and the amniotic fluid index was normal. 
Fasting state laboratory studies on the preterm pregnancy underwent fenofibrate treatment showed: plasma glucose 87 $\mathrm{mg} / \mathrm{dL}$ (normal range 70-110), serum triglyceride (TG) level of $414 \mathrm{mg} / \mathrm{dL}$ (normal range 50-179), thyroid-stimulating hormone (TSH) $2.12 \mathrm{ulU} / \mathrm{mL}$ (normal range 0.34-5.60) and ALT 29 $\mathrm{U} / \mathrm{L}$ (normal range 11-41). Laboratory studies were performed upon admission to the services, at 10 weeks of gestation and after two weeks of not using fenofibrate. At this time, gestational diabetes mellitus was diagnosed by an oral glucose tolerance test according to ADA criteria (7). The patient's serum fructosamine level was $272.7 \mu \mathrm{mol} / \mathrm{L}$ (normal range 205-285), the TG level was $3616 \mathrm{mg} / \mathrm{dL}$, amylase was $46 \mathrm{U} / \mathrm{L}$ (normal range 28-100) and lipase was $40.44 \mathrm{U} / \mathrm{L}$ (normal range 13-60). Type $\mathrm{V}$ hyperlipoproteinemia was diagnosed by lipoprotein electrophoresis according to the World Health Organization classification (8).

Although insulin therapy was not required for gestational diabetes, a single daily dose of NPH insulin (10 U) subcutaneously was started for the treatment of hypertriglyceridemia. A 1700 calorie lipid-poor diabetic diet was given to the patient. In the next few weeks of pregnancy, blood glucose did not require altering the dose of insulin and this dose was continued until the end of pregnancy with single daily NPH insulin 10 $U$ subcutaneously. There were no complications during treatment with insulin such as hypoglycemia. Total weight gain during pregnancy was $10 \mathrm{~kg}$. The patient's pregnancy course had not been complicated since her admission and she gave birth to a male infant by Caesarean section, weighing $3100 \mathrm{~g}$ at 38 weeks of gestation. The patient's clinical course is shown in Figure 1. Serum lipids were within normal limits in the infant (TG $94 \mathrm{mg} / \mathrm{dL}$ and total cholesterol $148 \mathrm{mg} / \mathrm{dl}$ (normal range: $150-200 \mathrm{mg} / \mathrm{dL})$ ).

By one month postpartum, the mother's serum TG had decreased to $299 \mathrm{mg} / \mathrm{dL}$ under dietary control without medication. Although her blood glucose level was normal, her triglyceride level was higher $(1317 \mathrm{mg} / \mathrm{dL})$ at three months postpartum and insulin therapy was started again.

\section{Discussion}

In normal individuals, the increase in plasma triglyceride level in pregnancy is modest, but in those who have deficien-

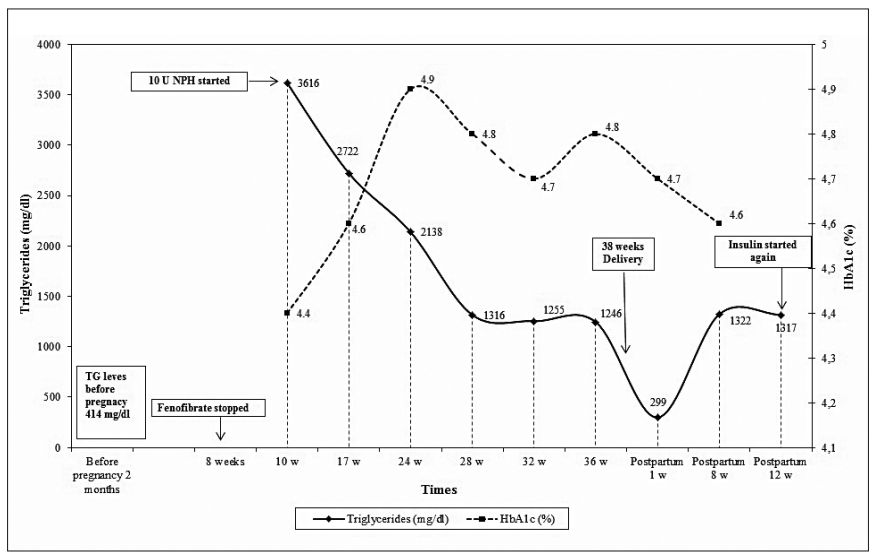

Figure 1. Changes in $\mathrm{HbA} 1 \mathrm{c}$ and plasma triglyceride levels cies in lipoprotein lipase or apolipoprotein C-II (i.e., familial hyperlipidemia syndrome type I, IV such as our patient, and V), this increased VLDL synthesis cannot be controlled and the result is severe hypertriglyceridemia (9). The exacerbation of triglyceride levels in familial hyperlipidemia during pregnancy is multifactorial. An increase in estrogen levels during pregnancy results in increased hepatic very low-density lipoprotein (VLDL) synthesis (3). Lipoprotein lipase activity is also reduced in pregnancy. Furthermore, human placental lactogen increases adipose tissue lipolysis and serum-free fatty acid levels, thus facilitating the availability of substrates to the liver for triglyceride synthesis. The combination of these phenomena a result in an increase in VLDL production coupled with reduced triglyceride clearance, leading to a net increase in serum triglyceride levels (4). Furthermore, secondary hypertriglyceridemia is associated with diabetes mellitus (usually with poor glycemic control). Mild hyperglycemia was also detected in our patient. We believe that gestational diabetes mellitus may be an additional result of hypertriglyceridemia in our patient.

Severe hypertriglyceridemia can lead to complications such as acute pancreatitis, preeclampsia, maternal and fetal complications. Therefore, patients with severe hyperlipidemia must be managed in order to avoid the associated complications. The main treatment modality for hypertriglyceridemia during pregnancy includes dietary restriction of fat, heparin and insulin, lipid-lowering medications (mainly fibrates) and parenteral feeding (10). Omega-3 fatty acids and plasmapheresis are effective and relatively harmless methods that can be used in pregnant women with severe hypertrigylceridemia and pancreatitis (10). However, plasmapheresis is not sponsored by the government in Turkey. Although most fibrate drug information sheets and formularies such as the Turkish National Formulary state that fibrates are contraindicated in pregnancy, there have been a few reports on the successful use of gemfibrozil to reduce the risk of developing hypertriglyceridemic pancreatitis during pregnancy with no side effects, particularly in the third trimester (10). Teratogenic effects are associated with fibrate therapy in pregnancy; therefore, alternative treatment strategies are required (11).

Although intravenous or intensive short-acting regular insulin therapy corrects severe hypertriglyceridemia especially complicated by acute pancreatitis in diabetic and non-diabetic patients, data regarding the use of single dose subcutaneous intermediate-acting NPH insulin for the management of severe hypertriglyceridemia in mildly hyperglycemic pregnant women has not been reported. The rationale for using insulin for the treatment of severe hypertriglyceridemia is based on the fact that insulin is a rapid and potent activator of lipoprotein lipase in the muscle and adipose tissue, most likely acting by increasing its synthesis (6). Insulin therapy may be an effective and safe treatment modality for severe hypertriglyceridemia in non-diabetic individuals. Data from a study by Jabbar et al. reported that a single dose injection of regular insulin immediately decreased the triglyceride level in non-diabetic patients with massive hypertriglyceridemia and thus reduced the risk of acute pancreatitis. However, their study showed that this therapy was unable to maintain the level below $1000 \mathrm{mg} /$ $\mathrm{dL}$, while long-term maintenance therapy could be provided 
by gemfibrozil (12). Rapid-acting insulin was not selected to avoid hypoglycemia and a single daily dose of NPH insulin $(0.15 \mathrm{lU} / \mathrm{kg}, 10 \mathrm{lU} /$ day) was started for the treatment of hypertriglyceridemia in our patient.

Here, we report a case of hypertriglyceridemia and mild hyperglycemia in a 33-year-old pregnant woman in whom successful treatment with merely single one time subcutaneous injection of intermediate-acting NPH insulin was accompanied by a reduction in the serum triglyceride level. We believe that patients with severe hypertriglyceridemia must be managed carefully in order to avoid complications. Because of the teratogenic effects associated with fibrate therapy in pregnancy, alternative treatment strategies such as insulin are required during pregnancy. Further studies are required to evaluate the effectiveness and safety of subcutaneously intermediateacting NPH insulin for the treatment of severe hypertriglyceridemia in non-diabetic pregnant women.

\section{Conflict of Interest}

No conflict of interest was declared by the authors.

\section{References}

1. Ma $Y$, Ooi TC, Liu MS, Zhang $H$, McPherson $R$, Edwards $A L$, et al. High frequency of mutations in the human lipoprotein lipase gene in pregnancy-induced chylomicronemia: possible association with apolipoprotein E2 isoform. Journal of lipid research 1994;35:1066-75.

2. McGladdery SH, Frohlich JJ. Lipoprotein lipase and apoE polymorphisms: relationship to hypertriglyceridemia during pregnancy. Journal of lipid research 2001;42:1905-12.
3. Fahraeus L, Larsson-Cohn U, Wallentin L. Plasma lipoproteins including high density lipoprotein subfractions during normal pregnancy. Obstetrics and gynecology 1985;66:468-72.

4. Herrera E, Lasuncion MA, Gomez-Coronado D, Aranda P, LopezLuna P, Maier I. Role of lipoprotein lipase activity on lipoprotein metabolism and the fate of circulating triglycerides in pregnancy. American journal of obstetrics and gynecology 1988;158:157583.

5. Hsia SH, Connelly PW, Hegele RA. Successful outcome in severe pregnancy-associated hyperlipemia: a case report and literature review. The American journal of the medical sciences 1995;309:213-8.

6. Eckel RH. Lipoprotein lipase. A multifunctional enzyme relevant to common metabolic diseases. The New England journal of medicine 1989;320:1060-8.

7. Standards of medical care in diabetes--2011. Diabetes care 2011;34:S11-61.

8. Beaumont JL, Carlson LA, Cooper GR, Fejfar Z, Fredrickson DS, Strasser T. Classification of hyperlipidaemias and hyperlipoproteinaemias. Bulletin of the World Health Organization 1970;43:891-915

9. Saravanan P, Blumenthal S, Anderson C, Stein R, Berkelhammer C. Plasma exchange for dramatic gestational hyperlipidemic pancreatitis. Journal of clinical gastroenterology 1996;22:295-8.

10. Al-Shali K, Wang J, Fellows F, Huff MW, Wolfe BM, Hegele RA. Successful pregnancy outcome in a patient with severe chylomicronemia due to compound heterozygosity for mutant lipoprotein lipase. Clinical biochemistry 2002;35:125-30.

11. Ujhazy E, Onderova E, Horakova M, Bencova E, Durisova M, Nosal R, et al. Teratological study of the hypolipidaemic drugs etofylline clofibrate (VULM) and fenofibrate in Swiss mice. Pharmacology \& toxicology 1989;64:286-90.

12. Jabbar MA, Zuhri-Yafi MI, Larrea J. Insulin therapy for a non-diabetic patient with severe hypertriglyceridemia. Journal of the American College of Nutrition 1998;17:458-61. 